\title{
An animal study of a novel tri-layer wound dressing material — non-woven fabric grafted with $N$-isopropyl acrylamide and gelatin
}

\author{
Feng-Huei Lin ${ }^{\mathrm{a}, *}$, Tim-Mo Chen ${ }^{\mathrm{b}}$, Ko-Shao Chen ${ }^{\mathrm{c}}$, Te-Hsing Wu ${ }^{\mathrm{d}}$, Chia-Chieh Chen ${ }^{\mathrm{d}}$ \\ ${ }^{a}$ Institute of Biomedical Engineering, College of Engineering and Medicine, National Taiwan University, Taipei, Taiwan \\ ${ }^{\mathrm{b}}$ Burn Center - Department of Surgery, Tri-service General Hospital, Taipei, Taiwan \\ ${ }^{\mathrm{c}}$ Tatung Institute of Technology, Taipei, Taiwan \\ ${ }^{\mathrm{d}}$ Institute of Nuclear Energy Research, P.O. Box 3-17, Lung-Tan, Taiwan
}

Received 20 July 1999; received in revised form 18 November 1999; accepted 23 November 1999

\begin{abstract}
A novel method of preparation of easily stripped-off temporary wound dressing material is described. A tri-layer membrane system for artificial skin is prepared in this study. In this process, the $N$-isopropyl acrylamide monomer is successfully grafted on the non-woven fabric by co-polymerization. It is initiated by plasma to activate the surface of the non-woven cloth. $N$-isopropyl acrylamide is then grafted onto the surface of the non-woven cloth by $\gamma$-ray irradiation. The last, but the most important, layer of a bovine gelatin with glycosaminoglycans (chondroitin-6-sulfate) is grafted by UV light, which serves as a matrix for the infiltration of fibroblasts, macrophages, lymphocytes, and capillaries derived from the wound bed.

The goal in this work is to provide a non-antigenic membrane closely resembling dermis in its anatomic structure and chemical composition, which would act as a scaffold inducing the synthesis of a new dermis. The following describes the construction and animal testing of this artificial skin when there is extensive damage. In the experiment, the specimens are divided into four groups: (1) control group without dressing material; (2) non-woven fabric; (3) non-woven fabric grafted with NIPAAm; and (4) non-woven fabric grafted with NIPAAm, bovine gelatin, and glycosaminoglycans from bottom to top in sequence.

Six weeks after the operation both, the control and non-woven fabric groups stayed in the proliferative phase where no epidermis or dermis structure could be traced in the section; however, the third group had healed completely in the maturation phase. The wound site had been totally recovered with normal dermis and epidermis structure around, but with the dressing material still staying on the wound site. In the group of the non-woven fabric grafted with NIPAAm, gelatin, and glycosaminoglycans, the wound had recovered to the final stage of maturation phase. The wound site had totally recovered at the 4 th week post operation. The dressing material of the group fell off automatically from the wound site without any damage to the skin after recovery. We believe the dressing material will have a great potential in medical application in the near future. (C) 2000 Elsevier Science S.A. All rights reserved.
\end{abstract}

Keywords: Artificial skin; N-isopropyl acrylamide; Tri-layer membrane

\section{Introduction}

Artificial skin is urgently needed to maintain the body temperature of a severely burned patient. Actual permanent replacement of skin by biomaterials is still a great clinical challenge. The treatment of extensively burned patients is a difficult clinical problem, not only because of the extent of the physiologic abnormalities caused by the burn itself, but also because of the small area of normal skin available to provide replacement of the large area of skin destruction. Such a replacement must take place if the patient is to sur-

\footnotetext{
* Corresponding author. Tel.: +886-2-23970800, ext. 1449; fax: +886-2-23940049.

E-mail address: double@ha.mc.ntu.edu.tw (F.-H. Lin)
}

vive the injury. There have been substantial improvements in the physiologic management of burn shock, infection, and metabolism which have considerably improved acute burn management. However, physiological replacement of destroyed skin has not kept pace with the improvement in systemic management, so that the burn illness is greatly complicated by the persistence of a large, open wound. If this wound is not promptly closed, malnutrition and bacterial invasion set the stage for extensive complications and a high mortality rate [1-3].

In order to reduce the extent and duration of an open wound, measures such as the use of allograft in moderate and large injuries, and temporary transplantation and immunosuppression for massive injuries, as well as technical innovations, such as autograft meshing, are used and have 
improved prognosis following an extensive burn [4]. Unfortunately, the full benefits of prompt excision of necrotic, burned tissue and immediate wound closure have not been realized, because there is no immediately available and physiologically acceptable replacement for extensive areas of destroyed skin [4,5].

We tried to prepare a tri-layer membrane system for artificial skin. The first layer is a dermal replacement layer, which is made of a three-dimensional porous cross-linked bovine gelatin with $\gamma$-ray irradiation. The second layer is a so-called connection layer that is poly- $N$-isopropylacrylamide (PNIPAAm) polymer. The third layer is a bovine gelatin with glycosaminoglycans (chondroitin-6-sulfate) that is manufactured with a controlled porosity and a well-defined degradation rate. The temporary epidermal substitute layer is made of synthetic non-woven cloth and functions to control moisture loss from the wound. The gelatin dermal replacement layer serves as a matrix for the infiltration of fibroblasts, macrophages, lymphocytes, and capillaries derived from the wound bed.

A major drawback in conventional dressing materials, mainly composed of gauze, is the adherence between the fibers of the gauze materials and the tissue. When fibers of the gauze are enclosed by the newly grown tissue as the wound becomes cured, the separation between the dressing material and the tissue becomes very difficult $[6,7]$. If the separation is not correctly performed, a secondary damage to the wound will be experienced and the recovery is also prolonged. The PNIPAAm connection layer adheres well with the tissue when the wound is in the moist condition. It will automatically and easily separate from the tissue once the wound is closed and in a dry condition. Epidermal substitute of the tri-layer membrane will serve as a protective film to prevent the wound from infection, fluid loss, and bacteria invasion as well.

The goal in this work is to provide a non-antigenic membrane closely resembling dermis in its anatomic structure and chemical composition, which would act as a biodegradable scaffolding inducing the synthesis of a 'neodermis'. The following describes the construction and animal testing of this artificial skin during extensive damage.

\section{Materials and experiments}

\subsection{Materials}

A widely used non-woven fabric of polyethylene is used as the substrate and this substrate can be pre-treated or surface-activated in a plasma chamber. The substrate is immersed in a solution of $N$-isopropyl acrylamide monomer (NIPAAm). An additive (vitamin B2) has to be added into the solution to prevent oxidation of the free radical in air. The substrate is then be exposed to irradiation by $\gamma$-rays so that NIPAAm can be grafted onto the surface of the sub- strate by co-polymerization. The radiation dose of $\gamma$-rays is preferably in the range of $10-100 \mathrm{kGy}$ and the irradiation time of about $10-20 \mathrm{~h}$. If UV light is used as the radiation source, the duration is preferably $10-90 \mathrm{~min}$ [8].

The surface modified non-woven fabric will be immersed into a gelatin solution of $16.7 \%$ concentration and then exposed to UV light for $90 \mathrm{~min}$. Finally, the treated non-woven fabric is soaked in the glycosaminoglycan solution for $2 \mathrm{~h}$ to form the dressing material [8-9].

In the experiment, the specimen were divided into four groups: (1) a control group without dressing material; (2) non-woven fabric; (3) non-woven fabric grafted with NIPAAm; and (4) non-woven fabric grafted with NIPAAm, bovine gelatin, and glycosaminoglycans from bottom to top in sequence.

\subsection{Animal experiment}

From a professional stock breeder, 20 mature New Zealand male rabbits with an average weight of $3.0 \pm 0.5 \mathrm{~kg}$ were obtained. After at least 2 weeks in quarantine and examination by a veterinary surgeon, they were operated upon. All surgical procedures were performed under sterile circumstances and followed by 3 days of pre-operative antibiotic protection with ampicillin $20 \%$ (Alfasan, $0.1 \mathrm{ml} / 2.5 \mathrm{~kg}$ ). The guidelines of the Ethical Committee of National Taiwan University for the care and use of laboratory animals were observed [10]. Rabbits were anesthetized with subcutaneous medetetomidine (Dormitor) $0.3 \mathrm{mg} / \mathrm{kg}$, ketamine (Ketalar) $20 \mathrm{mg} / \mathrm{kg}$, and diazepam (Diapam) $0.15 \mathrm{mg} / \mathrm{kg}$. A circular defect of $5 \mathrm{~cm}$ diameter was created at the central area of dorsum along the dorsimesad. The area around the defect of each rabbit was shaved and scrubbed before operation. Through a short incision, the epidermis, dermis and superficial fascia were monoblocally excised from the dorsal area of the experimental animal. The prepared dressing materials were then covered onto the hole thus created, and fixed with 4-0 nylon. The rabbits were scarified after 2, 4, and 6 weeks of operation. There were three rabbits for each group in every experimental period.

\subsection{Histology}

The entire specimens with surrounding tissue were collected and fixed in $4 \%$ buffered formalin solution. Specimens were processed through serial alcohol solution, toluene and full-faced section embedding in paraffin. The procedure used is the celloidin-paraffin double-embedding method, which takes $96 \mathrm{~h}$ on an automatic specimen processor. Then the specimens were impregnated with paraffin wax in the usual manner and embedded. Transverse sections, about $5 \mu \mathrm{m}$ in thickness, were obtained using a microtome. Sections were stained with hematoxylin and eosin (HE) for optical microscopic examination. 


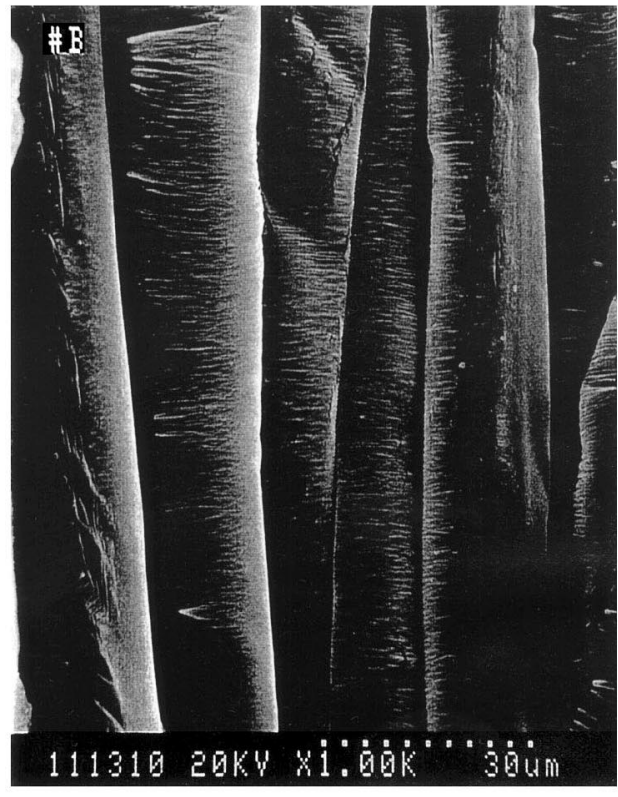

(a)

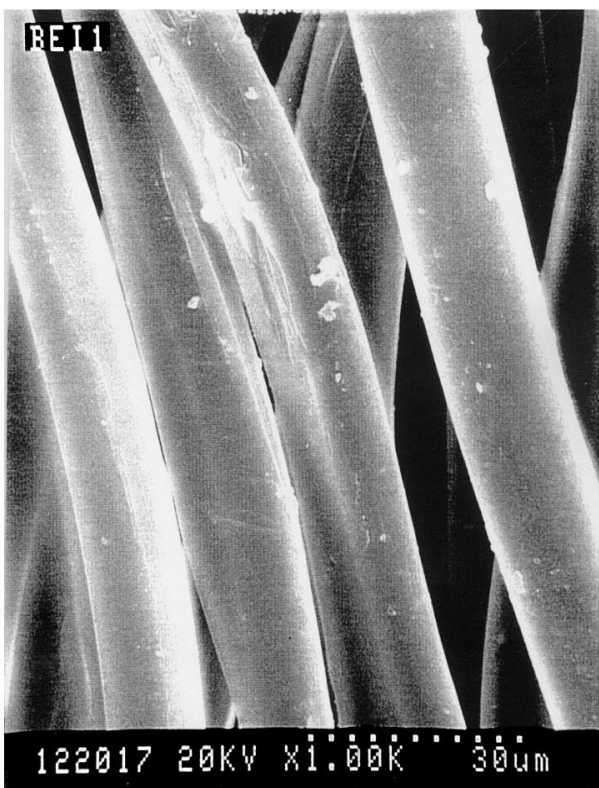

(b)

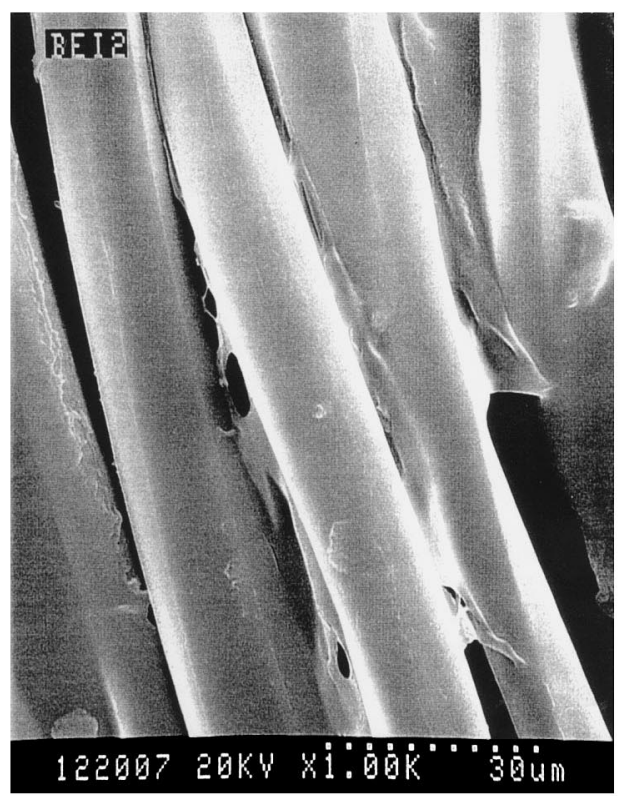

(c)

Fig. 1. The morphology of the dressing materials: (a) non-woven fabric without any treatment; (b) grafted with NIPAAm; and (c) gelatin grafted on the NIPAAm.

\section{Results}

\subsection{Morphology of the materials}

Fig. 1 shows the morphology of the dressing materials. Fig. 1a is the non-woven fabric without any treatment, which shows a rough surface. After grafting with NIPAAm, the non-woven fabric exhibits a more smooth surface (Fig. 1b). Once gelatin and glycosaminoglycans topped on the NIPAAM, the non-woven fabric forms a tri-layer dressing material with a non-antigenic membrane closely resembling dermis in its anatomic structure and chemical composition, which will induce the dermis regeneration (Fig. 1c).

\subsection{Histological observations}

When an injury extends to tissue deep in the epidermis, due to accidental lacerations or surgical incisions, the repair process is more complex than epidermal healing and scar formation results. Generally, the healing process is completed through four-stages [11]: the inflammatory phase; the migratory phase; the proliferative phase; and the maturation 


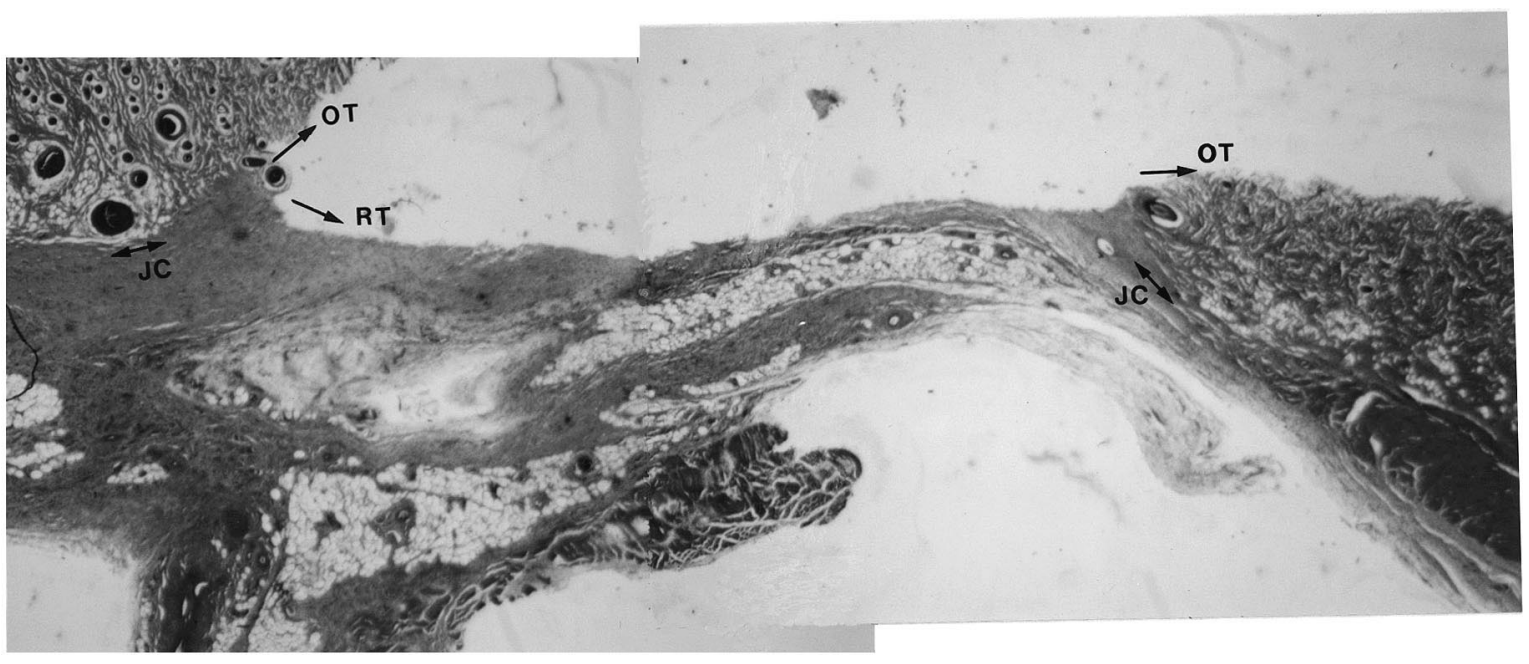

Fig. 2. The non-woven fabric is supposed to be in the migratory phase after 2 weeks of operation. OT, original skin tissue; RT, recovery skin tissue; and $\mathrm{JC}$, junction.

phase. We will use these four phases to describe the histological observations during recovery.

\subsubsection{Histological observation after 2 weeks of operation}

In the control group, without a covering of any dressing material, the wound is in the so-called inflammatory phase. A blood clot forms in the wound and loosely unites the wound edges, epithelial cells begin migrating across the wound, vasodilatation and increased permeability of blood vessels deliver neutrophils and monocytes that phagocytize microbes, and mesenchymal cells develop into fibroblasts.

The group of non-woven fabric is supposed to be in the migratory phase (Fig. 2). The clot becomes a scab and epithelial cells migrate beneath the scab to bridge the wound, fibroblasts migrate along fibrin threads and begin synthesiz- ing scar tissue, and damaged blood vessels begin to re-grow. During this phase, tissue filling the wound is the so-called granulation tissue.

In the group of non-woven fabric grafted with NIPAAm, the wound is in the stage of proliferative phase (Fig. 3). An extensive growth of epithelial cells beneath the scab, the deposition of collagenous fibers in random patterns by fibroblasts, and the continued growth of blood vessels are all observed in the picture.

For the group of non-woven fabric grafted with NIPAAm, gelatin, and glycosaminoglycans, there is little difference in the healing stage of the upper and lower parts of the wound site (Fig. 4). Upper part of the wound site has been in the final phase (maturation phase) and the scab sloughs-off once the epidermis is restored to normal thickness, collagenous fibers

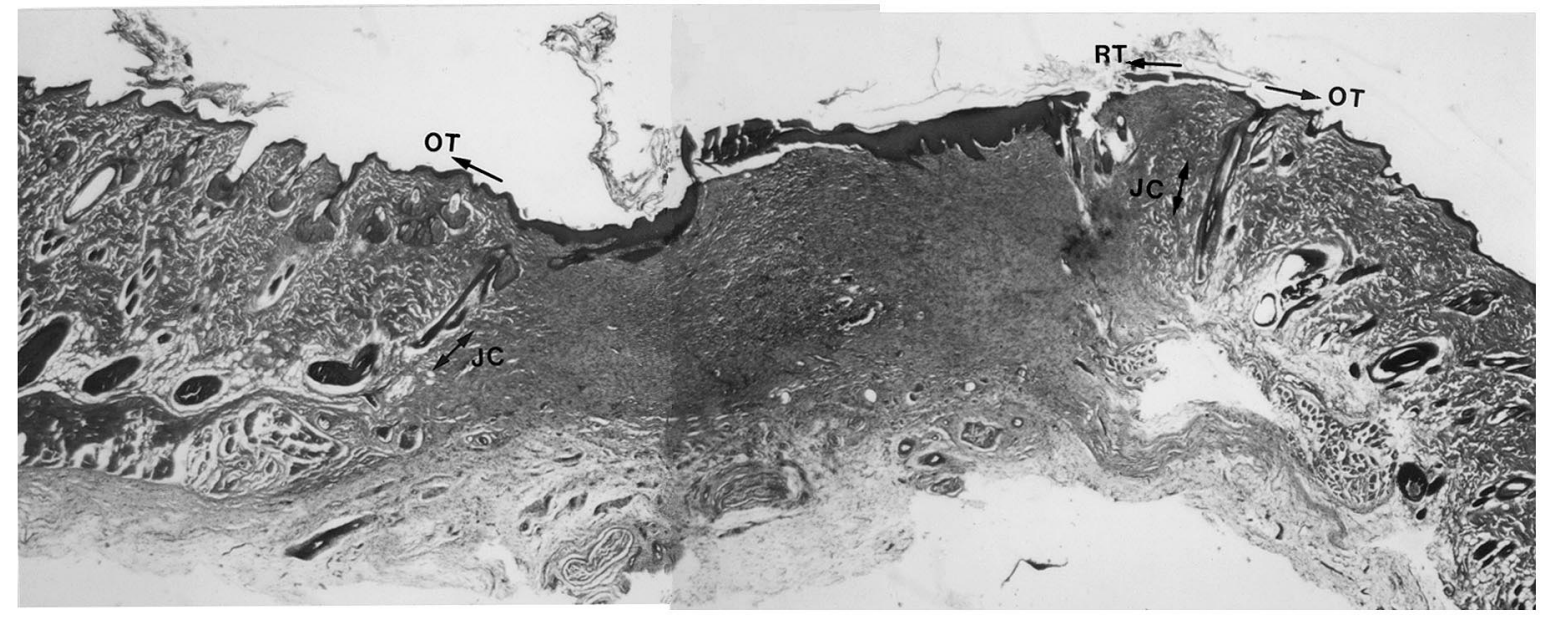

Fig. 3. Two weeks post-operation, the non-woven fabric grafted with NIPAAm is in the proliferative phase. OT, original skin tissue; RT, recovery skin tissue; and JC, junction. 


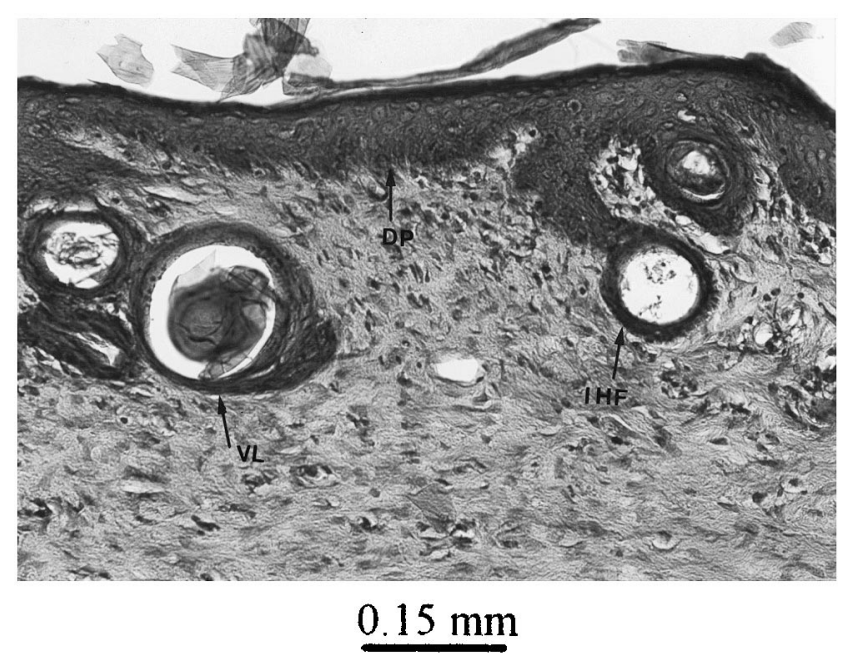

Fig. 4. Two weeks post-operation, the non-woven fabric grafted with NIPAAm and gelatin is in the maturation phase at the upper part and in the proliferative phase at the lower part. VL, blood vessel; and IHF, burgeon of hair follicle.

become more organized, fibroblasts begin to disappear, and blood vessels are restored to normal. The lower part of the wound site is still in the proliferation phase.

\subsubsection{Histological observation of 4 weeks post-operation}

In the controlled group, the wound was slowly healing and supposed to be in the migratory phase. Sporadic and loose fibrous tissue filled the wound site.

In the group of non-woven fabric without NIPAAm or gelatin grafted, the wound is identified in the stage of prolif- erative phase, but no epidermis or dermis structure has been traced in the section.

Four weeks post-operation, the group of non-woven fabric grafted with NIPAAm has been completely stepped into the final stage of proliferative phase (Fig. 5). The dermal papillae, hair follicle and sudoriferous (sweat) gland have been developed.

In the group of the non-woven fabric grafted with NIPAAm, gelatin, and glycosaminoglycans, the wound has recovered to the final stage of the maturation phase (Fig. 6). The wound site has totally recovered with normal dermis and epidermis structure around. The dressing material of the group has fallen off automatically from the wound site without any damage to the skin after recovery.

\subsubsection{Histological observation 6 weeks after dressing}

Six weeks after the operation both, the controlled and non-woven fabric groups stayed in the proliferative phase, where no epidermis or dermis structure has been traced in the section.

Six weeks post-operation, the group of non-woven fabric with NIPAAm grafted has been healed completely in the maturation phase. The wound site has been totally recovered with normal dermis and epidermis structure around, but the dressing material still stayed upon the wound site.

\section{Discussions}

Wound dressing includes a wide variety of wound care management devices, drugs, and biological materials. They may vary from simple skin bandages or ointments to

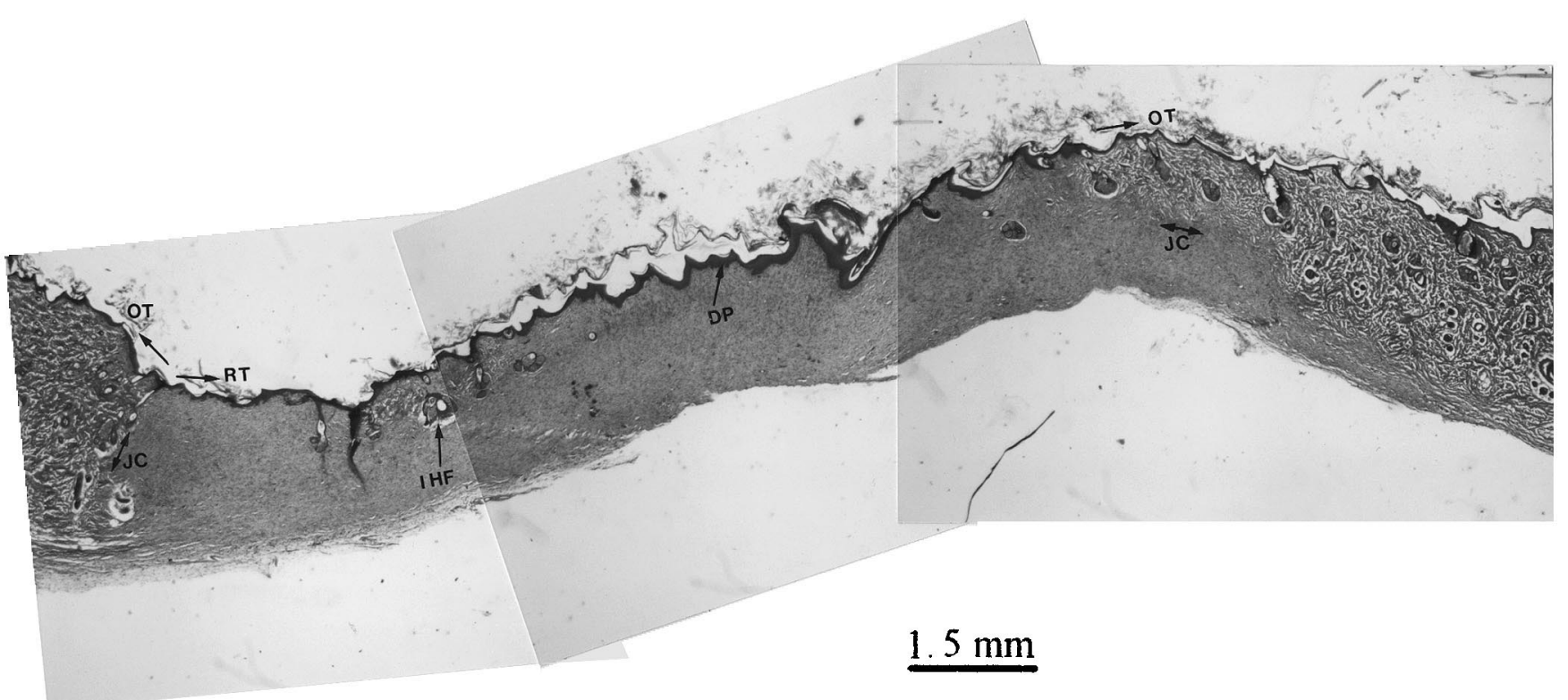

Fig. 5. Four weeks post-operation, non-woven fabric grafted with NIPAAm has been in the final stage of proliferative phase. OT, original skin tissue; RT, recovery skin tissue; JC, junction; IHF, burgeon of hair follicle; and DP, dermal papllia. 


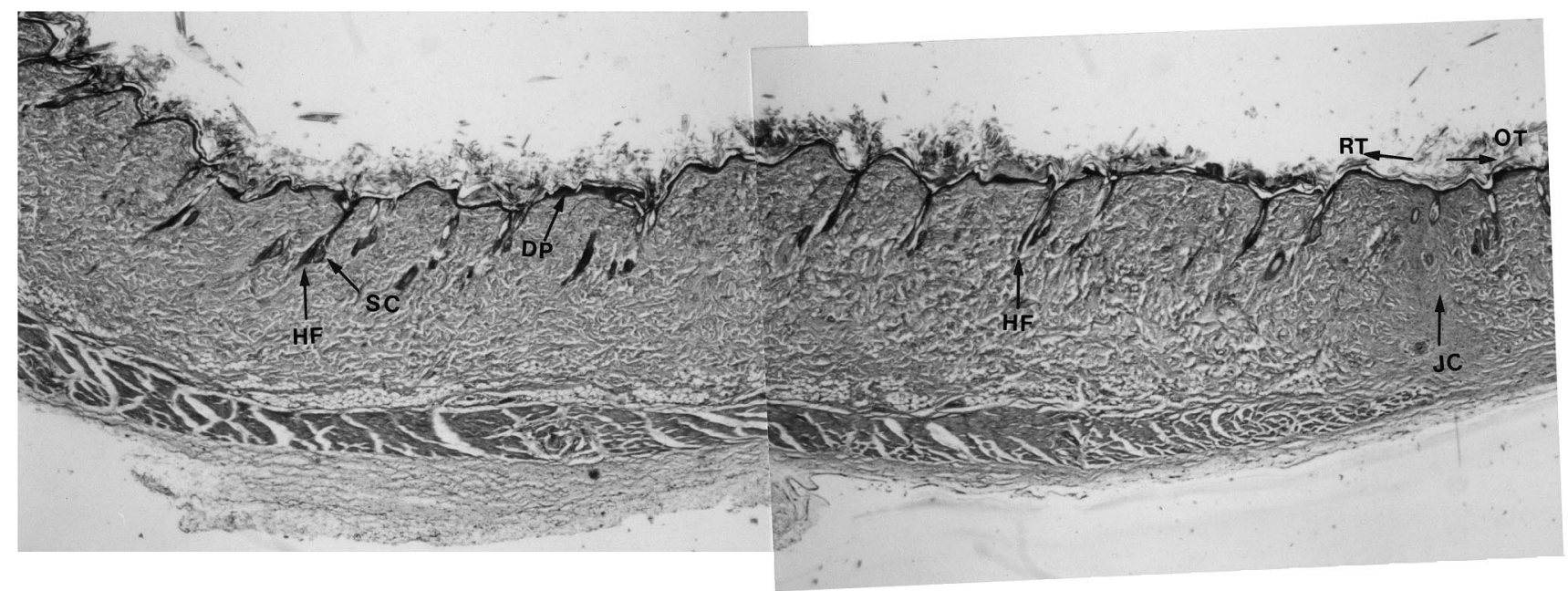

Fig. 6. In the group of non-woven fabric grafted with NIPAAm, geltain, and glycosaminoglycans the wound has recovered to the final stage of maturation phase after 4 weeks of operation. OT, original skin tissue; RT, recovery skin tissue; JC, junction; HF, hair follicle; DP, dermal papllia; and SC, sebaceous gland.

full-thickness skin transplants. These dressings could be further classified as temporary wound closures, which include allografts, xenografts, and synthetic or biological materials, and permanent wound closure, which includes autografts, cultured epithelial cells, microskin grafting, and the like $[4,6,12]$. In the study, the non-woven fabric without coating and the fabric grafted with NIPAAm can be classified into, for example, the skin bandages which may prevent the wound site from further infection or fluid loss. However, the two dressings do not have the ability to induce new dermis or epidermis ingrowth, which delays the healing process.

From the histological observation, the non-woven fabric grafted with NIPAAm, gelatin, and glycosaminoglycans adhered till recovery to the final stage of maturation phase and fell off automatically from the wound site without any damage to the skin after recovery at the 4 th week post-operation. The dressing used in the study is a completely synthetic tri-layer membrane manufactured using native biological raw materials. The dermal analog is a porous structure made of bovine hide gelatin, covalently linked to chondroitin 6-sulfate. An appropriate pore size is critical to allow the optimal ingrowth of native fibroblasts and endothelial cells. The temporary outer layer, or epidermal analog, is a non-woven fabric to be replaced 21 days with an ultra-thin autoepidermal graft, which is widely meshed. Water flux across this membrane is the same as that across normal epidermis. The gelatin-glycosaminoglycans dermal analog layer provides a three-dimensional architecture for normal dermis approach. The layer is completely biodegradable after 30 days. After serving as a scaffold for the ingrowth of self-fibroblasts and endothelial cells, a normal dermis-like layer will be formed $[3,4,13]$.

The dressing material applied to, and covering, the wounded area as a result of burning or scratching must be capable of absorbing the exuded liquid from the wounded area $[6,14]$. In addition, this dressing material also serves as an excellent barrier, which provides an intact environment such that the wounded tissue can be prevented from being infected by external bacteria or virus while the wounded tissue is recovering to its original condition. The connection layer of the tri-layer dressing is NIPAAm, which is a temperature-sensitive polymer. It has a lower critical solution temperature (LCST) of about $36-37^{\circ} \mathrm{C}$. The temperature of wound site is higher than this transition temperature, because of histamine being released from local inflammation. When applied onto the surface of a wound site, the fabric will absorb the exuded fluid and will be attached to the surface. The NIPAAm will become hydrophobic and fall off automatically once the wound site has healed.

The ideal skin replacement should have characteristics including immediate availability, a prolonged shelflife, the ability to replace both, the epidermal and dermal components of normal skin, near-normal mechanical behavior and cosmetic appearance, normal antibacterial defense, effective wound healing after injury, and growth with patient growth [15-16].

\section{Conclusion}

The tri-layer wound dressing has been used successfully in the treatment of extensive skin injury. The histological changes in, and around, the dressing on the wound bed were over a period that extended from 7 to 42 days. The process of wound healing and repair was divided into four phases that were recognized by certain histological features. Occasionally, eosinophils or giant cells were observed. Their appearance did not correlate with clinical problems. These cellular infiltrates appear to be entirely healed and repaired under such circumstances. No immune reaction that correlated 
with rejection of the dressing was observed. The dressing falls off automatically without any damage, once the wound site healed completely.

\section{Acknowledgements}

The authors would like to express their gratitude to the National Science Council of ROC for their financial support to the research. We also wish to thank the Nuclear Research Center of ROC for their technical support in $\gamma$-ray radiation in the study.

\section{References}

[1] D. Michaeli, M. McPherson, Immunologic study of artificial skin used in the treatment of thermal injuries, J. Burn Care Rehabil. 11 (1) (1990) 21-26.

[2] I.V. Yannas, J.F. Burke, Design of an artificial skin 1. Basic design principles, J. Biomed. Mater. Res. 14 (1980) 107-115.

[3] R.L. Sheridan, M. Hefarty, R.G. Tompkins, J.F. Burke, Artificial skin in massive burns - results to ten years, Eur J. Plast. Surg. 17 (1994) 91-93.

[4] V. Glaser, US skin replacement market could heat up, Bio/Technology 13 (9) (1995) 933-940.

[5] B. Coulomb, L. Friteau, J. Baruch, J. Guilbaud, B. Chretien-Marquet, J. Glicenstein, C. L-Decoster, E. Bell, L. Dubertret, Advantage of the presence of living dermal fibroblasts within in vitro reconstructed skin for grafting in humans, Plast. Reconstr. Surg. 101 (7) (1998) 1891-1903.

[6] J.F. Burke, Observations on the development and clinical use of artificial skin: an attempt to employ regeneration rather than scar formation in wound healing, Jpn. J. Surg. 17 (1987) 431-438.

[7] I. Feller, D. Tholen, R.G. Cornell, Improvements in burn care, 1965-1979, J. Am. Med. Assoc. 244 (1980) 2074-2080.

[8] T.H. Wu, F.H. Lin, C.C. Chen, K.S. Chen, The preparation of wound dressing materials by radiation irradiated grafting, Chemistry 55 (3) (1997) 51-58.

[9] S.F. Lin, K.S. Chen, The easily stripped wound dressing, Chinese J. Biomed. Eng. 3 (1993) 219-221.

[10] F.H. Lin, C.J. Liao, H.C. Liu, K.S. Chen, J.S. Sun, Behavior of fetal rat osteoblast cultured in vitro on the DP-bioglass substratum, Mater. Chem. Phys. 49 (1997) 270-276.

[11] R. Stern, M. McPherson, M.T. Longaker, Histologic study of artificial skin used in the treatment of full-thickness thermal injury, J. Burn Care Rehabil. 23 (6) (1997) 501-504.

[12] D.P. Orgill, I.V. Yannas, Design of artificial skin, J. Biomed. Mater. Res. 39 (4) (1998) 531-535.

[13] C.C. Compton, C.E. Butler, I.V. Yannas, G. Warland, D.P. Orgill, Organized skin structure is regenerated in vivo from collagen-GAG matrices seeded with autologous keratinecytes 110 (6) (1998) 908926.

[14] C. Lorenz, A. Petracic, H.P. Hohl, L. Wessel, K.L. Waag, Early wound closure and early reconstruction, Burns 23 (6) (1997) 505508.

[15] K. Soejima, M. Nozaki, K. Sasaki, M. Takeuchi, N. Negishi, Reconstruction of burn deformity using artificial dermis combined with thin split-skin grafting 23 (6) (1997) 501-504.

[16] W.H. Eaglstein, V. Falanga, Tissue engineering and the development of Apligraf, a human skin equivalent, 19(5) (1997) 894-905. 